\title{
A key link between jasmonic acid signaling and auxin biosynthesis
}

\author{
HE YuBing ${ }^{1} \&$ ZHAO Yunde ${ }^{2 *}$ \\ ${ }^{1}$ National Key Laboratory of Crop Genetic Improvement and National Centre of Plant Gene Research, Huazhong Agricultural University, \\ Wuhan 430070, China; \\ ${ }^{2}$ Section of Cell and Developmental Biology, University of California San Diego, La Jolla, California 92093-0116, USA
}

Received January 15, 2015; accepted February 5, 2015; published online February 9, 2015

Citation: He YB, Zhao Y. A key link between jasmonic acid signaling and auxin biosynthesis. Sci China Life Sci, 2015, 58: 311-312, doi: $10.1007 / \mathrm{s} 11427-015-4821-8$

A research team led by Xiang ChengBin from School of Life Sciences, University of Science and Technology of China discovered a molecular basis for crosstalk between jasmonic acid (JA) and auxin, two important plant hormones. Their findings were published in a recent issue of Nature Communication [1].

Auxin is the plant growth hormone that plays an essential role in almost every aspect of plant growth and development including primary root elongation and lateral root initiation [2]. Disruption of auxin biosynthesis, transport, or signal transduction by genetic or pharmacological approaches leads to severe defects in root development [2]. Auxin overproduction mutants such as yuccal-D and surl produce more lateral roots whereas some auxin signaling mutants fail to initiate any lateral roots in Arabidopsis [2]. On the other hand, JA is generally considered one of the plant stress hormones that regulate plant responses to various abiotic and biotic stresses. JA is also known as a regulator for several developmental processes including lateral root development under normal growth conditions. Arabidopsis seedlings treated with JA have short primary roots and more lateral roots [3,4], a phenotype that has also been observed in auxin-treated plants and auxin overproduction mutants. Therefore, it has long been recognized that $\mathrm{JA} /$ auxin crosstalk probably plays an important role in root development.

One of the potential mechanisms of JA/auxin crosstalk is that JA controls plant developmental processes by modulat-

*Corresponding author (email: yundezhao@ucsd.edu) ing auxin biosynthesis [3,4]. It has been well documented that plants treated with JA produce more auxin. Moreover, some auxin biosynthetic genes were identified from genetic screens for JA-resistant mutants, suggesting that auxin biosynthesis is required for normal JA responses [3,4]. However, the molecular mechanisms by which JA regulates auxin biosynthesis have remained elusive for decades. A breakthrough occurred recently when Cai et al. identified a JA-inducible transcription factor ERF109 that directly binds to the promoters of auxin biosynthetic genes, thus providing a key molecular link for the JA/auxin crosstalk during lateral root development [1].

Research often leads to unexpected findings and the discovery of the roles of ERF109 in JA/auxin crosstalk is no exception. Xiang ChengBin and his colleagues were interested in understanding why one of their drought tolerance mutants, edtl (enhanced drought tolerance 1), had a more extensive root system [5]. After analyzing the root transcriptional profiles of both $e d t 1$ and wild type, they discovered that ERF109 was dramatically induced in the edt $1 \mathrm{mu}-$ tant. The fact that ERF109 is a known JA-inducible gene and that $e d t l$ has a root system similar to that of JA treated plants. This observation led to their hypothesis that ERF109 probably plays an important role in JA/auxin crosstalk.

Indeed, the transcription level of ERF109 was greatly increased in both the shoot and root, especially in lateral root primordia upon JA treatments. Moreover, the JA receptor COI1 was also required for the induction of ERF109 by JA. The functions of ERF109 were further studied using genetic approaches. Overexpression of ERF109 led to shorter pri- 
mary roots and more lateral roots whereas erf109 mutants had fewer lateral root primordia per unit root length. More importantly, erf109 mutants were partially insensitive to JA treatments during lateral root development. The genetic data suggest that ERF109 is a positive regulator of JA signaling. Furthermore, ERF109 has a profound effect on controlling auxin concentrations in Arabidopsis plants. Plants overexpressing ERF109 produced more auxin and displayed the characteristic auxin overproduction phenotypes including more and longer root hairs, and longer hypocotyls.

ERF109 directly regulates auxin biosynthesis during lateral root development. The expression levels of ASAI and $Y U C 2$, two known auxin biosynthetic genes, were downregulated in erf109 and up-regulated in ERF109 overexpression lines. That ERF109 directly binds to the GCCboxes of ASAI and YUC2 promoters was supported by results generated from assays of yeast one hybrid, electrophoretic mobility shift, and chromatin immunoprecipitation. In conclusion, Cai et al. [1] presented convincing evidence that ERF109 serves as a key molecular link for the crosstalk between JA signaling and local auxin biosynthesis. The findings can be used to optimize a plant root system, thus enabling us to develop more drought tolerant plants.

1 Cai XT, Xu P, Zhao PX, Liu R, Yu LH, Xiang CB. Arabidopsis ERF109 mediates cross-talk between jasmonic acid and auxin biosynthesis during lateral root formation. Nat Commun, 2014, 5: 5833

2 Zhao Y. Auxin biosynthesis. Arabidopsis Book, 2014, 12: e0173

3 Hentrich M, Bottcher C, Duchting P, Cheng Y, Zhao Y, Berkowitz O, Masle J, Medina J, Pollmann S. The jasmonic acid signaling pathway is linked to auxin homeostasis through the modulation of YUCCA8 and YUCCA9 gene expression. Plant J, 2013, 74: 626-637

4 Sun J, Xu Y, Ye S, Jiang H, Chen Q, Liu F, Zhou W, Chen R, Li X, Tietz O, Wu X, Cohen JD, Palme K, Li C. Arabidopsis ASA1 is important for jasmonate-mediated regulation of auxin biosynthesis and transport during lateral root formation. Plant Cell, 2009, 21: 1495-1511

5 Yu H, Chen X, Hong YY, Wang Y, Xu P, Ke SD, Liu HY, Zhu JK, Oliver DJ, Xiang CB. Activated expression of an Arabidopsis HD-START protein confers drought tolerance with improved root system and reduced stomatal density. Plant Cell, 2008, 20: 1134-1151

Open Access This article is distributed under the terms of the Creative Commons Attribution License which permits any use, distribution, and reproduction in any medium, provided the original author(s) and source are credited. 